\title{
Chemical Polymerization of Aniline Using Para-toluene Sulphonic Acid
}

\author{
R. Ratheesh and K. Viswanathan \\ Department of Physics, Karpagam University, Coimbatore -641021, Tamilnadu
}

\begin{abstract}
Polyaniline doped with hydrochloric acid $(\mathrm{HCl})$ was prepared by chemical oxidative polymerization of aniline (ANI) in aqueous medium with ammonium peroxy disulphate as an oxidant. The deprotonation of the PANI: HCl samples were performed by stirring the PANI: HCl powder (emeraldine salt) in 0.1 M aqueous solution of ammonium hydroxide for 24 hours at room temperature, thus obtaining the polymer in the emeraldine base form. The redoping of the PANI (emeraldine base) with para-toluene sulphonic acid was performed by mixing emeraldine base (EB) with para- toluene sulphonic acid (PTSA) to obtain the required doping levels $(Y=0.5, Y=0.3, Y=0.1)$, known as the molar ratio of TSA to phenyl-nitrogen repeat units $(S / N)$.The prepared samples were characterized by Fourier transform-infrared (FT-IR) spectroscopy, Ultraviolet-Visible (UV-VIS) spectroscopy and X-ray diffraction technique.The prepared samples were pressed into pellets, and DC electrical conductivity was measured, using four-probe method. The FT-IR peaks obtained in the samples are in good agreement with the reported peaks in literature. UV-VIS spectroscopy shows the various electron transitions present in the polyaniline samples. The crystallinity of the samples was studied in detail by X-ray diffraction technique.The conductivity shows an increase with increase in temperature, for all the three samples.
\end{abstract}

Key Words:Polyaniline, chemical synthesis, polymerization, emeraldine, FT-IR,XRD, conductivity studies.

\section{Introduction}

Polyaniline is the oxidative polymeric product of aniline under acidic conditions and has been known since 1862 as aniline black [1]. Among all the conducting polymers, polyaniline (PANI) is the most promising polymer due to its easy synthesis, controllable electrical conductivity in doped state, good environmental stability and simple non-redox doping by protonic acids. Polyaniline is a typical phenylene based polymer having a chemically flexible $-\mathrm{NH}-$ group in the polymer chain flanked either side by phenylene ring [2]. The protonation, deprotonation and various other pysico-chemical properties of polyaniline are due to the presence of this $-\mathrm{NH}-$ group [2].

It is well known that PANI exists in three different oxidation states such as leucoemeraldine, emeraldine, and pernigraniline.Among these only polyemeraldine is electrically conductive. The electronic transport properties of PANI can be changed by doping either by electrochemically or chemically with some anions [3].In the recent years, PANI has been one of the most extensively investigated conducting polymers due to its electronic, electrochemical, and optical properties. In addition, PANI has thermal stability, particularly in the conducting emeraldine salt form and is a candidate for potential commercial application, such as in lightemitting diodes, lightweight battery electrodes, sensors, electro-optics, electromagnetic shielding materials, biochemical capacitors, and anticorrosion coating $[4,5,6,7,8]$.

In this paper, the chemical synthesis and characterization of polyaniline-paratoluene sulphonic acid salts are described. Aniline was polymerized with paratoluene sulphonic acid by chemical oxidative polymerization using ammonium peroxy disulphate as an oxidising agent. The polymer samples were characterized by infrared, UV-Vis, XRD and conductivity measurements.

\section{Experimental}

Synthesis of emeraldine salt is done as explained in [9].

\subsection{Emeraldine Base Doped With Para-Toluene Sulphonic Acid (Ptsa)}

In the present study, para- toluene sulphonic acid (PTSA) was used as a dopant. The PANI - (PTSA)y was prepared in three different doping levels [The doping level is defined as $\mathrm{Y}=$ (mole acid)/ (mole PANI)]. The PANI - (PTSA)Y was prepared by a thorough mixing of emeraldine base PANI with para toluene sulponic acid (PTSA) using a glass mortar and pestle to obtain required doping levels, known as the molar ratio of PTSA to phenyl - nitrogen repeat units $(\mathrm{S} / \mathrm{N})$. 
1. The PANI-(PTSA) 0.5 was prepared by a thorough mixing of $0.012 \mathrm{M}$ emeraldine base PANI with $0.006 \mathrm{M}$ para-toluene sulphonic acid (PTSA) using a glass mortar and pestle to obtain the required doping level Y= 0.5. This is the maximum level of doping.

2. The PANI-(PTSA) 0.3 was prepared by a thorough mixing of $0.012 \mathrm{M}$ emeraldine base PANI with $0.0036 \mathrm{M}$ para-tolune sulphonic acid (PTSA) using a glass mortar and pestle to obtain the required doping level $\mathrm{y}=$ 0.3 .

3. The PANI-(PTSA) 0.1 was prepared by a thorough mixing of $0.012 \mathrm{M}$ emeraldine base PANI with $0.0012 \mathrm{M}$ para-toluene sulphonic acid (PTSA) using a glass mortar and pestle to obtain the required doping level $\mathrm{Y}=0.1$.

For all studies, the doped PANI with $\mathrm{Y}=0.5, \mathrm{Y}=0.3$ and $\mathrm{Y}=0.1$ are used.

The prepared emeraldine salt powder was made into a pellet for conductivity studies using four probe apparatus. Electrical conductivity was calculated using the following equation [7]

$$
\rho=\frac{\rho_{0}}{G_{7}\left(\frac{W}{S}\right)}
$$

Where $\boldsymbol{G}_{\mathbf{7}}\left(\frac{\boldsymbol{W}}{\boldsymbol{S}}\right)$ is a correction divisor [it is a function of thickness of the sample as well as probe-spacing and equals to $\left(\frac{2 S}{W}\right) \log _{e}$ ] and $\rho_{0}=\frac{I}{2 \pi S}$ where I, V,W and S are current (A), voltage (V), thickness of the film (cm) and probe-spacing $(\mathrm{cm})$ respectively.

The conductivity was measured at different temperatures (from room temperature to $110^{\circ} \mathrm{C}$ ). The current (I) was kept constant during the experiment.

\subsection{UV-Visible Spectroscopy}

\section{Results And Discussion}

The UV-Vis spectra of PANI-(PTSA $)_{x}(\mathrm{x}=0.5,0.3,0.1)$, are shown in Fig.1, Fig.2 and Fig.3 respectively.The absorption bands at $300 \mathrm{~nm}$ in Fig.1, $294 \mathrm{~nm}$ in Fig.2 and $300 \mathrm{~nm}$ in Fig.3are assigned to the $\pi$ $\rightarrow \pi^{*}$ electronic transition of the phenyl rings in the conducting polymer backbone. The absorption peak at 630 $\mathrm{nm}$ in Fig.1, $644 \mathrm{~nm}$ in Fig.2 and $604 \mathrm{~nm}$ in Fig.3 are due to the inter-band charge transfer associated with excitation of benzoid to quinoid moieties (exciton transition) [10].The absorption peak at $776 \mathrm{~nm}$ in Fig.1, 828 $\mathrm{nm}$ in Fig.2 and $790 \mathrm{~nm}$ in Fig.3 are assigned to the polaron band [11], the characteristic peak observed in the protonated polyaniline representing the conducting emeraldine salt phase of the polymer.
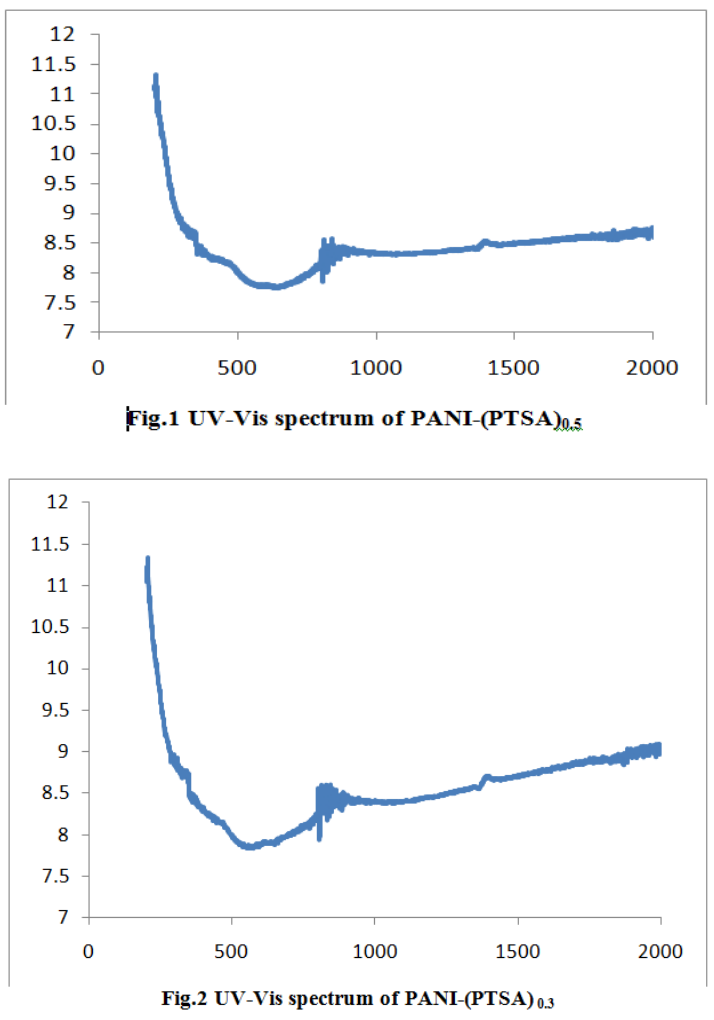

www.iosrjournals.org 


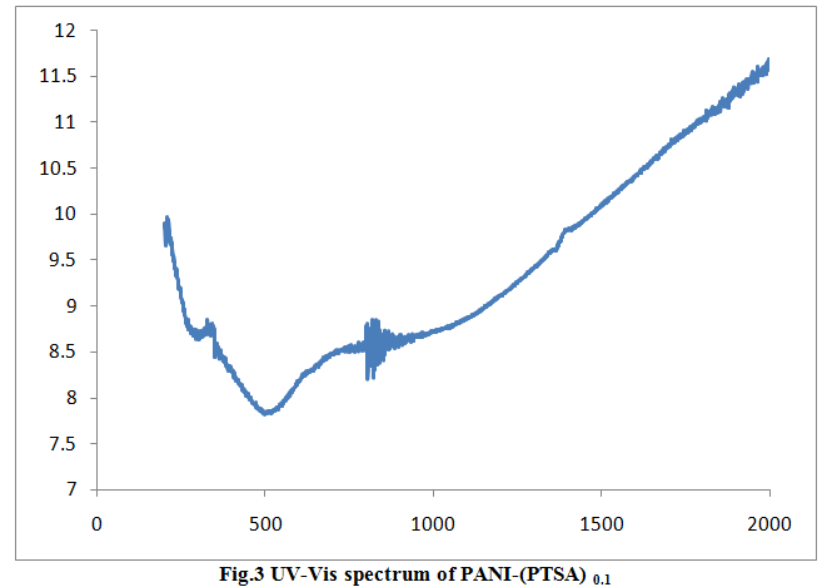

\subsection{FTIR Spectra}

The FTIR Spectrum of para toluene sulphonic acid (PTSA) doped polyaniline $(\mathrm{y}=0.5,0.3,0.1)$ are shown in Figs.4, 5 and 6 respectively.The peaks at $1560.30 \mathrm{~cm}^{-1}$ in all the three spectra show the presence of quinoid and the peaks at $1488.94 \mathrm{~cm}^{-1}$ in Fig.4, at $1490.87 \mathrm{~cm}^{-1}$ in Figs. $5 \& 6$ show the presence of benzoid ring, thereby indicating the oxidation state of emeraldine salt PANI[12].A strong band observed at $1139.85 \mathrm{~cm}^{-1}$ in Fig.4, at $1140 \mathrm{~cm}^{-1}$ in Fig.5 and at $1143.71 \mathrm{~cm}^{-1}$ in Fig.6, described as the electronic-like band, is considered to be a measure of the degree of delocalization of electrons and thus it is characterized peak of PANI[13]. The band at $1569.95 \mathrm{~cm}^{-1}$ in all the three spectra is attributed to a $\mathrm{C}-\mathrm{N}$ stretching in the neighborhood of quinoid ring. The $1298 \mathrm{~cm}^{-1}$ band in Fig. 4 \& 5 and at $1301.86 \mathrm{~cm}^{-1}$ in Fig. 6 are assigned to the $\mathrm{C}-\mathrm{N}$ stretch of secondary aromatic amine, whereas the band at $1419.51 \mathrm{~cm}^{-1}$ in Fig. 4 and at $1419.59 \mathrm{~cm}^{-1}$ in Figs. 5 \& 6 are assigned to the aromatic $\mathrm{C}-\mathrm{H}$ in plane bending modes. Out-of-plane bending deformation of $\mathrm{C}-\mathrm{H}$ is observed at $565.1 \mathrm{~cm}^{-1}$ for PANI-(PTSA) $0_{0.5}$, at $567.03 \mathrm{~cm}^{-1}$ for PANI-(PTSA) $0_{0.3}$ and at $568.96 \mathrm{~cm}^{-1}$ for PANI-(PTSA) $)_{0.1}$.A band observed at $798.47 \mathrm{~cm}^{-1}$ in Fig. 4 and at $800.4 \mathrm{~cm}^{-1}$ in Figs. $5 \& 6$ can be ascribed to the paradisubstituted aromatic rings indicating polymer formation. The peaks at $1029.92 \mathrm{~cm}^{-1}$ in Fig. 4 and at $1031.85 \mathrm{~cm}^{-1}$ in Figs. $5 \& 6$ can be assigned due to $\mathrm{SO}_{3}{ }^{-}$group of the dopant PTSA.

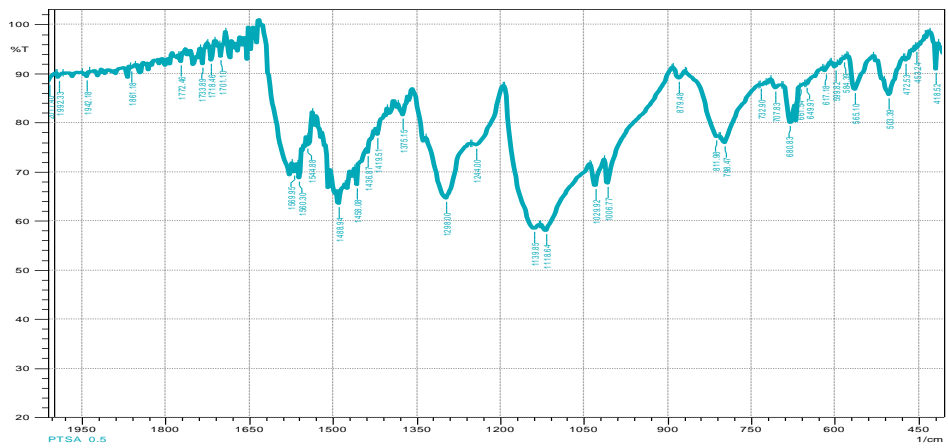

Fig.4FTIR spectrum of PANI-(PTSA) $)_{0.5}$

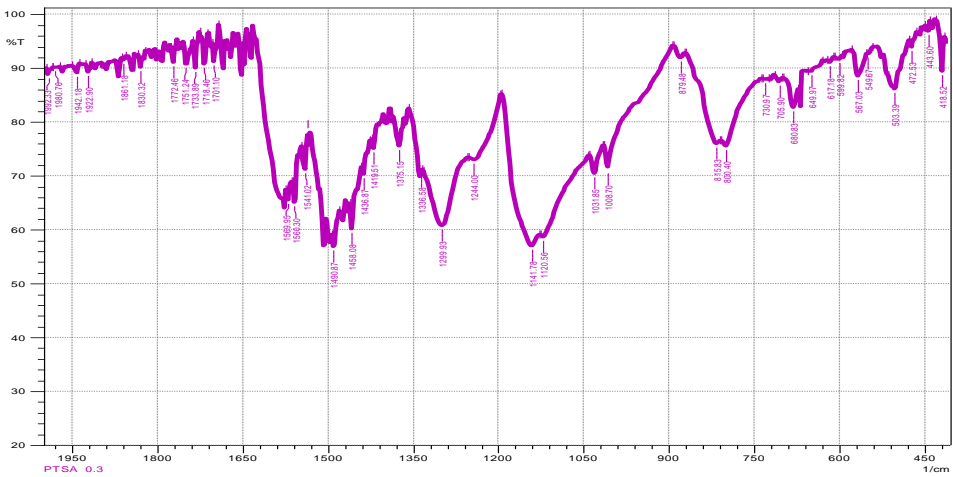

Fig.5 FTIR spectrum of PANI-(PTSA) $)_{0.3}$ 


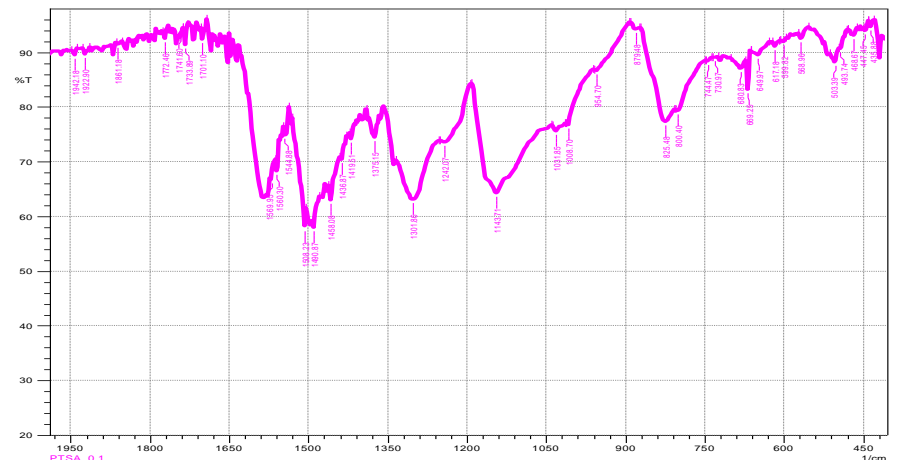

Fig.6 FTIR spectrum of PANI-(PTSA) ${ }_{0.1}$

\subsection{X-ray Diffraction}

The X-ray diffraction pattern of the para-toluene sulphonic acid (PTSA) doped polyaniline (PANI) at three different doping levels $(\mathrm{y}=0.5,0.3$ and 0.1$)$ are shown in fig.7,8 and 9 respectively. The diffraction patterns obtained in this work for PANI-(PTSA) $)_{0.5}$, PANI-(PTSA) $)_{0.3}$ and PANI-(PTSA) $)_{0.1}$ shows the conducting emeraldine salt phase of the polymer. The X-ray diffraction patterns of the three doped polymers indicate a broad angle asymmetric scattering peaks from $10^{\circ}$ to $30^{\circ}$. Few diffraction patterns obtained in this work can be considered as typical of poly emeraldine salt (ES).This is because the protonation levels in three samples studied are close to 0.5 , i.e., the value expected for ideal poly emeraldine salt (ES). This diffractogram showsa set of three reflections with the higher relative intensity, for $2 \theta \approx 16.461,21.670$ and $27.343^{\circ}$. Also, three distinct reflections of lower intensity can be distinguished from the diffractograms, for $2 \theta \approx 6.7,5.337$ and $4.947^{\circ}$. The appearance of peaks in PANI-(PTSA) $)_{\mathrm{Y}}$ indicates significant crystallization of PANI on protonation indicating the conducting emeraldine phase of the polymer. The PTSA doped PANI has a clear diffraction peak at $2 \theta$ $=25.146^{\circ}$ for PANI-(PTSA $)_{0.5}, \quad 2 \theta=16.461^{\circ}$ for PANI-(PTSA $)_{0.3}$ and $2 \theta=19.115^{\circ}$ for PANI-(PTSA $)_{0.1}$ in the background of amorphous shoulder, indicating that some part of the PANI sub chains become rigid and well ordered . Doped polyaniline is considered as the polymer cation-anion complex here, polyaniline could be treated as a poly-cation while TSA acts as an anion that allows an increase in interchain packing and thus higher structural order,which is similar to the accepted results for $\mathrm{HCl}$ doped polyaniline indicating the doped state of the polyaniline. In addition to this, it is observed that the intensities of the peak around $2 \theta=15.104^{\circ}$ for PANI(PTSA ) $)_{0.5}, 2 \theta=16.466^{\circ}$ for PANI-(PTSA ) 0.3 and $2 \theta=16.474^{\circ}$ for PANI-(PTSA ) $)_{0.1}$ are strongly increased by increasing the protonation ratio indicating that the conductivity of p-TSA doped polyaniline increases with increase in the doping level.

SN 0.5

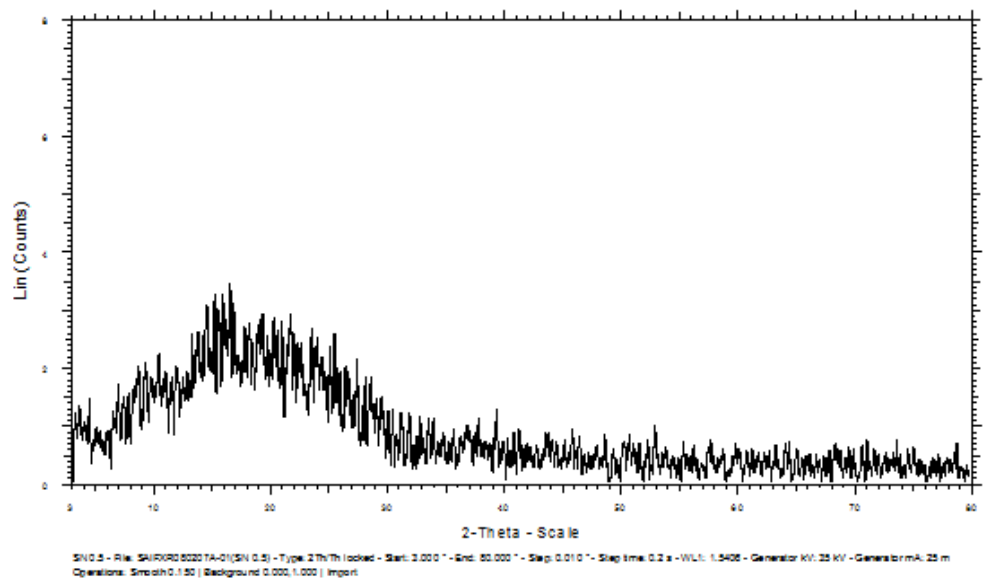

Fig.7 X-ray diffraction pattern of PANI-(PTSA) $)_{0.5}$ 


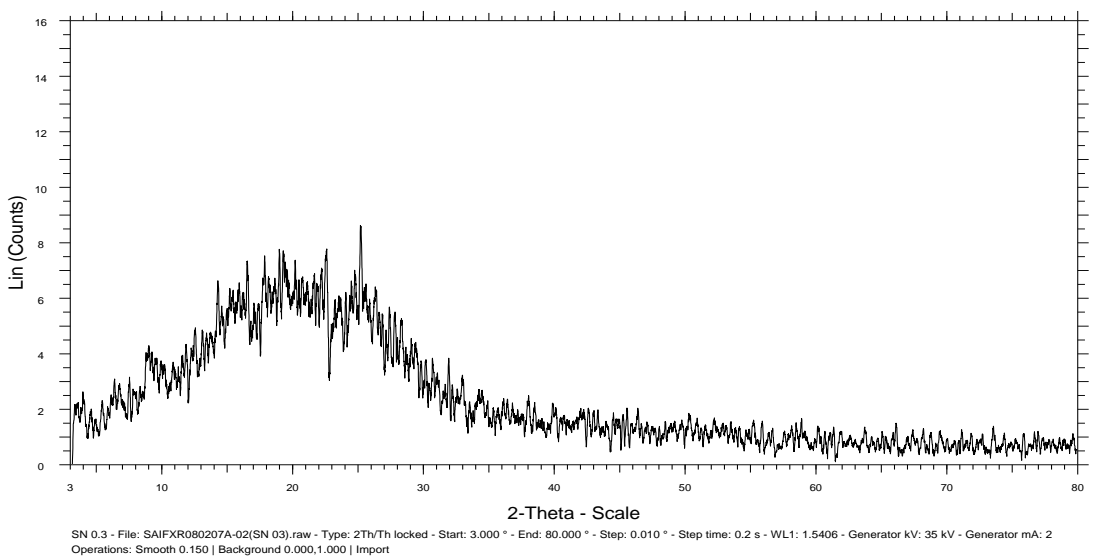

Fig.8 X-ray diffraction pattern of PANI-(PTSA) $)_{0.3}$

SN 0.1

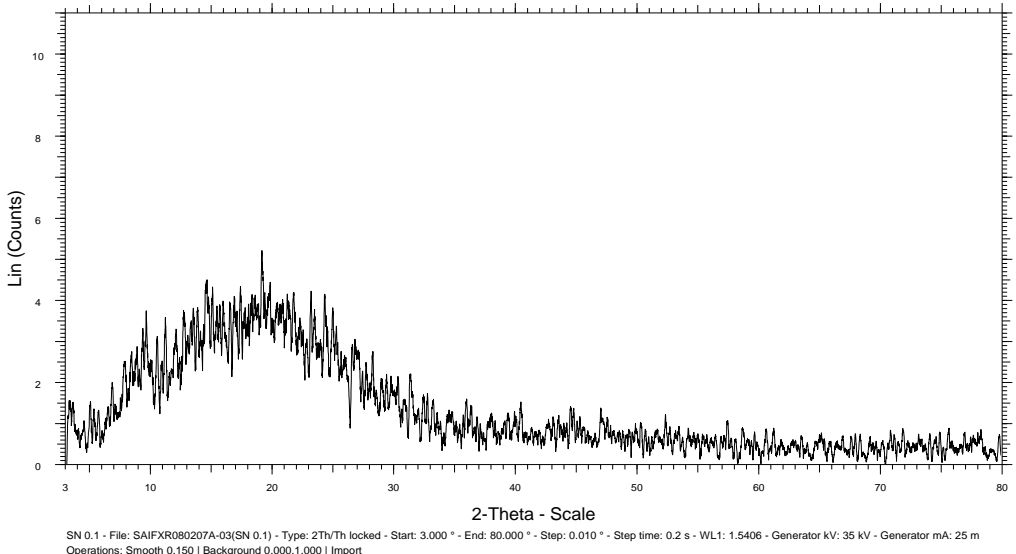

Fig.9 X-ray diffraction pattern of PANI-(PTSA) 0.1

\subsection{Electrical conductivity}

The change in conductivity with increasing temperature of PANI-(PTSA) $)_{0.5}$, PANI-(PTSA) 0.3 and PANI-(PTSA) $)_{0.1}$ are shown in Figs.10,11 and 12 respectively, and the values are summarized in Tables 1, 2 and 3 respectively. The temperature dependence of conductivity gives important information about the electrical conduction mechanism in the doped polyaniline. The para-toluene sulphonic acid (PTSA) doped polyaniline (PANI) at different doping levels $(\mathrm{y}=0.5,0.3,0.1)$ behaves like a semiconductor and its electrical conductivity increases with increase in temperature. On comparison with the standard values of electrical conductivity for traditional semiconductors, it is observed that the initial electrical conductivity of the PTSA doped PANI is well within the semiconductor region.

The graphs for all the three different doping levels show that the conductivity increases constantly with increasing temperature. The initial electrical conductivity was observed around $10^{-2} \mathrm{~S} \mathrm{~cm}^{-1}$ for PANI- (PTSA) 0.5 , $10^{-3} \mathrm{~S} \mathrm{~cm}^{-1}$ for PANI- (PTSA) $)_{0.3}$ and $10^{-5} \mathrm{~S} \mathrm{~cm}^{-1}$ for PANI- (PTSA) $0_{0.1}$ respectively at $35^{\circ} \mathrm{C}$. However, the temperature dependence of electrical conductivity of PTSA doped PANI (in all the three doping levels) increases with increase of temperature, which suggests its thermal stability in the presence of oxygen.

The temperature dependence of resistivity was fitted to Arrhenius type equation and measured values were plotted logarithmically as a function of reciprocal of temperature. The fig.13, 14 and 15 shows the variation of $\log \rho$ with inverse temperature of PANI-(PTSA) $)_{0.5}$, PANI- (PTSA) 0.3 andPANI- (PTSA) 0.1 respectively. 
Table: 1 Conductivity of PANI-(PTSA) $)_{0.5}$

\begin{tabular}{|l|l|l|l|l|l|l|l|}
\hline $\begin{array}{l}\text { S. } \\
\text { No }\end{array}$ & $\begin{array}{l}\text { Temp } \\
\mathrm{T}^{\circ} \mathrm{C}\end{array}$ & $\begin{array}{l}\text { Voltage volt } \\
\mathrm{x} 10^{-3}\end{array}$ & $\begin{array}{l}\text { Temp } \\
\mathrm{T} \mathrm{K}\end{array}$ & $\begin{array}{l}\text { Resistivity } \\
\text { ohm }-\mathrm{cm}\end{array}$ & $\begin{array}{l}\text { conductivit } \\
\text { y S/cm }\end{array}$ & $\begin{array}{l}1 / \mathrm{T} \text { K } \\
\text { x } 0^{-3}\end{array}$ & Log $\rho$ \\
\hline 1 & 35 & 18.06 & 308 & 19.4757 & 0.05135 & 3.2468 & 1.28949 \\
\hline 2 & 40 & 17.35 & 313 & 18.7101 & 0.05345 & 3.1949 & 1.27208 \\
\hline 3 & 45 & 16.60 & 318 & 17.9013 & 0.05586 & 3.1447 & 1.25288 \\
\hline 4 & 50 & 15.95 & 323 & 17.2003 & 0.05814 & 3.0960 & 1.23554 \\
\hline 5 & 55 & 15.32 & 328 & 16.5209 & 0.06053 & 3.0488 & 1.21803 \\
\hline 6 & 60 & 14.75 & 333 & 15.9062 & 0.06287 & 3.0030 & 1.20157 \\
\hline 7 & 65 & 14.26 & 338 & 15.3778 & 0.06503 & 2.9586 & 1.18689 \\
\hline 8 & 70 & 13.78 & 343 & 14.8602 & 0.06729 & 2.9155 & 1.17202 \\
\hline 9 & 75 & 13.35 & 348 & 14.3965 & 0.06946 & 2.8736 & 1.15826 \\
\hline 10 & 80 & 12.92 & 353 & 13.9328 & 0.07177 & 2.8329 & 1.14404 \\
\hline 11 & 85 & 12.45 & 358 & 13.4259 & 0.07448 & 2.7933 & 1.12794 \\
\hline 12 & 90 & 12.19 & 363 & 13.1456 & 0.07607 & 2.7548 & 1.11878 \\
\hline 13 & 95 & 11.87 & 368 & 12.8005 & 0.07812 & 2.7174 & 1.10723 \\
\hline 14 & 100 & 11.60 & 373 & 12.5093 & 0.07994 & 2.6810 & 1.09723 \\
\hline 15 & 105 & 11.37 & 378 & 12.2613 & 0.08156 & 2.6455 & 1.08854 \\
\hline 16 & 110 & 11.09 & 383 & 11.9593 & 0.08362 & 2.6110 & 1.07771 \\
\hline
\end{tabular}

Table: 2 Conductivity of PANI-(PTSA) $)_{\mathbf{0 . 3}}$

\begin{tabular}{|l|l|l|l|l|l|l|l|}
\hline S.No & $\begin{array}{l}\text { Temp } \\
\left.\mathrm{T}^{\circ} \mathrm{C}\right)\end{array}$ & $\begin{array}{l}\text { Voltage volt } \\
\times 10^{-3}\end{array}$ & $\begin{array}{l}\text { Temp } \\
\mathrm{T} \mathrm{K}\end{array}$ & $\begin{array}{l}\text { Resistivity } \\
\text { ohm -cm } \\
\mathrm{x} 10^{3}\end{array}$ & $\begin{array}{l}\text { conductivity } \\
\text { S/cm }\end{array}$ & $\begin{array}{l}1 / \mathrm{T} \text { K } \\
\times 10^{-3}\end{array}$ & Log $\rho$ \\
\hline 1 & 35 & 10.78 & 308 & 0.31657 & 0.003159 & 3.2468 & 2.5005 \\
\hline 2 & 40 & 10.27 & 313 & 0.29773 & 0.003359 & 3.1949 & 2.4738 \\
\hline 3 & 45 & 9.78 & 318 & 0.28352 & 0.003527 & 3.1447 & 2.4526 \\
\hline 4 & 50 & 9.30 & 323 & 0.26961 & 0.003709 & 3.0960 & 2.4307 \\
\hline 5 & 55 & 8.93 & 328 & 0.25888 & 0.003863 & 3.0488 & 2.4131 \\
\hline 6 & 60 & 8.61 & 333 & 0.24961 & 0.004006 & 3.0030 & 2.3973 \\
\hline 7 & 65 & 8.28 & 338 & 0.24004 & 0.004166 & 2.9586 & 2.3803 \\
\hline 8 & 70 & 8.01 & 343 & 0.23221 & 0.004306 & 2.9155 & 2.3659 \\
\hline 9 & 75 & 7.78 & 348 & 0.22554 & 0.004433 & 2.8736 & 2.3532 \\
\hline 10 & 80 & 7.63 & 353 & 0.22120 & 0.004521 & 2.8329 & 2.3448 \\
\hline 11 & 85 & 7.42 & 358 & 0.21511 & 0.004649 & 2.7933 & 2.3327 \\
\hline 12 & 90 & 7.25 & 363 & 0.21018 & 0.004759 & 2.7548 & 2.3226 \\
\hline 13 & 95 & 7.07 & 368 & 0.20496 & 0.004879 & 2.7174 & 2.3117 \\
\hline
\end{tabular}

Table: 3 Conductivity of PANI-(PTSA) $)_{0.1}$

\begin{tabular}{|l|l|l|l|l|l|l|l|}
\hline S.No & $\begin{array}{l}\text { Temp } \\
\left.\mathrm{T}^{\circ} \mathrm{C}\right)\end{array}$ & $\begin{array}{l}\text { Voltage volt } \\
\mathrm{x} 10^{-3}\end{array}$ & $\begin{array}{l}\text { Temp } \\
\mathrm{T} \mathrm{K}\end{array}$ & $\begin{array}{l}\text { Resistivity } \\
\text { ohm }-\mathrm{cm} \times 10^{3}\end{array}$ & $\begin{array}{l}\text { conductivity } \\
\text { S/cm } \times 10^{-3}\end{array}$ & $\begin{array}{l}1 / \mathrm{T} \\
\times 10^{-3}\end{array}$ & $\begin{array}{l}\text { Log } \rho \\
1\end{array}$ \\
35 & 36.20 & 308 & 25.9168 & 0.0386 & 3.2468 & 4.4136 \\
\hline 2 & 40 & 33.50 & 313 & 23.9838 & 0.0417 & 3.1949 & 4.3799 \\
\hline 3 & 45 & 31.00 & 318 & 22.1940 & 0.0451 & 3.1447 & 4.3462 \\
\hline 4 & 50 & 28.60 & 323 & 20.4757 & 0.0488 & 3.0960 & 4.3112 \\
\hline 5 & 55 & 26.80 & 328 & 19.1870 & 0.0521 & 3.0488 & 4.283 \\
\hline 6 & 60 & 24.80 & 333 & 17.7552 & 0.0563 & 3.0030 & 4.2493 \\
\hline 7 & 65 & 23.20 & 338 & 16.6097 & 0.0602 & 2.9586 & 4.2204 \\
\hline 8 & 70 & 21.90 & 343 & 15.6790 & 0.0638 & 2.9155 & 4.1953 \\
\hline 9 & 75 & 20.30 & 348 & 14.5335 & 0.0688 & 2.8736 & 4.1624 \\
\hline 10 & 80 & 19.30 & 353 & 13.8175 & 0.0724 & 2.8329 & 4.1404 \\
\hline 11 & 85 & 18.10 & 358 & 12.9584 & 0.0772 & 2.7933 & 4.1126 \\
\hline 12 & 90 & 16.80 & 363 & 12.0277 & 0.0831 & 2.7548 & 4.0802 \\
\hline 13 & 95 & 16.00 & 368 & 11.4550 & 0.0873 & 2.7174 & 4.059 \\
\hline 14 & 100 & 15.10 & 373 & 10.8106 & 0.0925 & 2.6810 & 4.0339 \\
\hline
\end{tabular}




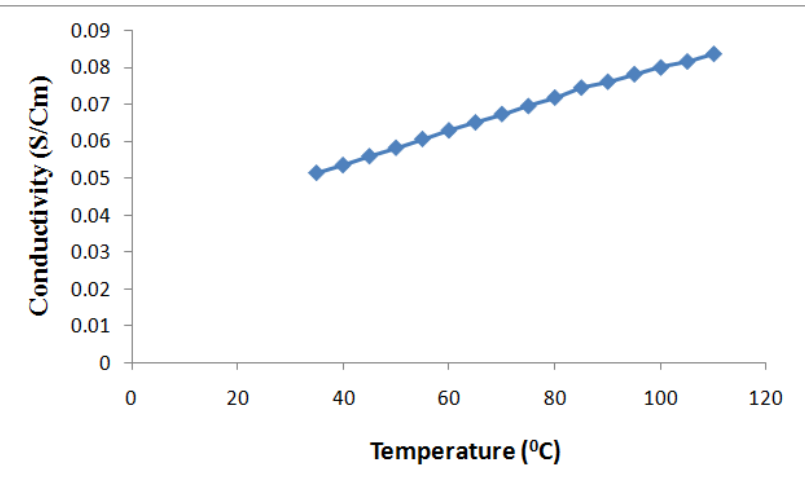

Fig.10. Change in conductivity with temperature of PANI-(PTSA) 0.5

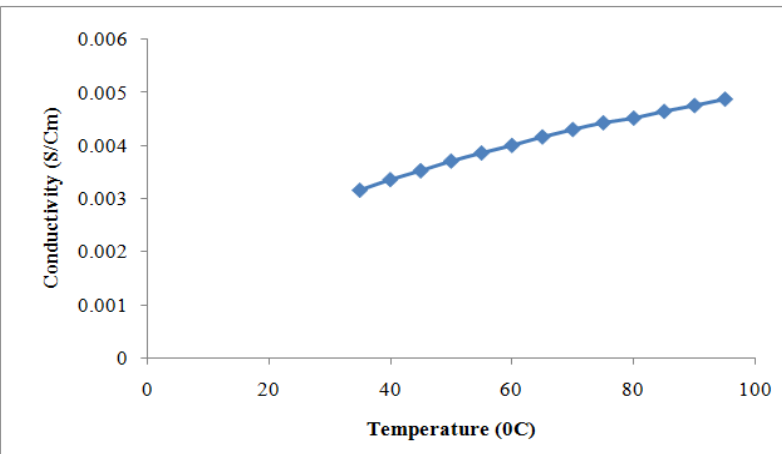

Fig.11. Change in conductivity with temperature of PANI-(PTSA) 0.3

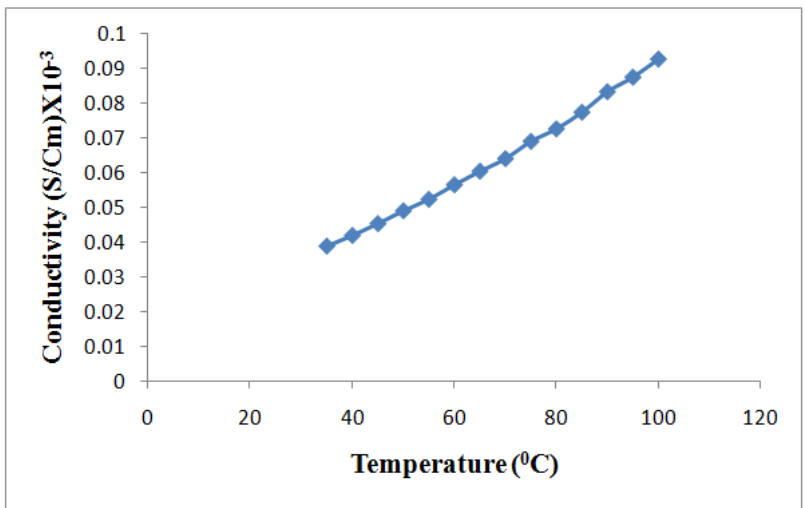

Fig.12. Change in conductivity with temperature of PANI-(PTSA) $)_{0.1}$

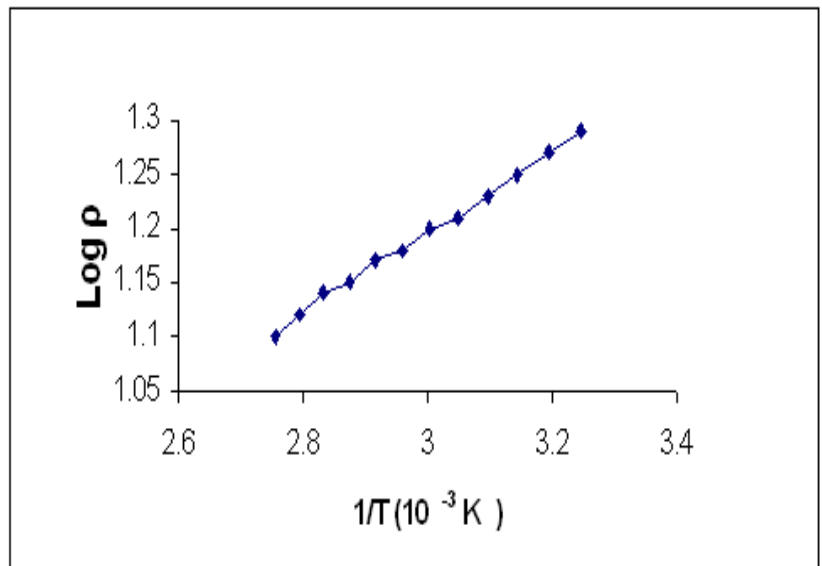

Fig.13 . Change in logarithmic resistivity with inverse temperature of PANI-(PTSA) $)_{0.5}$ 


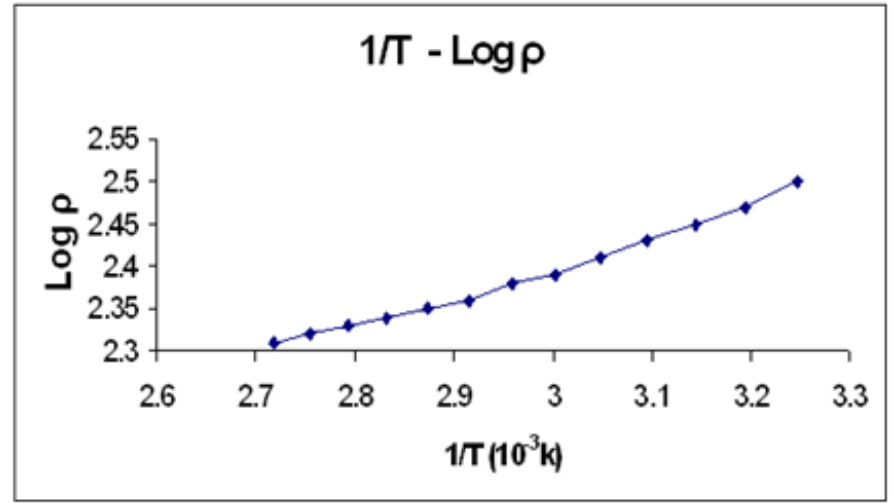

Fig. 14: Change in logarithmic resistivity with inverse temperature of PANI-(PTSA) ${ }_{0.3}$

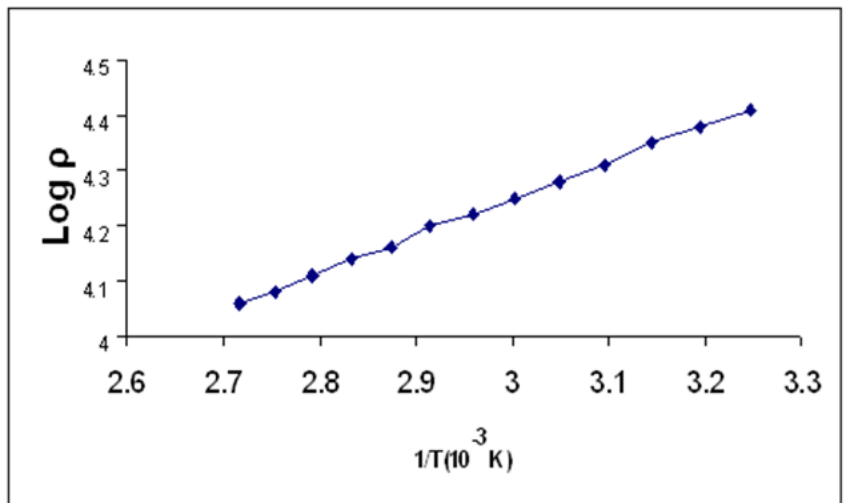

Fig.15 Change in logarithmic resistivity with inverse temperature of PANI-(PTSA) $)_{0.1}$

\section{Conclusions}

Polyaniline powders were synthesized by the chemical polymerization of aniline using ammonium peroxy disulphate in aqueous acid solution $(\mathrm{HCl})$. This green polymer powder (emeraldine salt) was converted in to non conducting dark blue emeraldine base by protonation withNH $\mathrm{H}_{4} \mathrm{OH}$. The redoping of the emeraldine base with para-toluene sulphonic acid (PTSA) was performed by mixing emeraldine base with p-TSA to obtain the required doping levels. The chemically synthesized polyaniline-PTSA salts were characterized by XRD, FTIR and UV-Vis spectroscopic techniques. The temperature dependent of electrical conductivity were determined using four probe apparatus. The conclusions arrived from the studies are as follows.

The $\pi \rightarrow \pi^{*}$ electronic transition of the phenyl rings in theconducting polymer backbone and the absorption peak due to the inter-band charge transfer associated with excitation of benzoid to quinoid moieties are observed from the UV spectral studies. The characteristic peak observed in the protonated polyaniline representing the conducting emeraldine salt phase of the polymer.

The FTIR Spectrum of the three samples of para-toluene sulphonic acid (PTSA) doped polyaniline exhibit the presence of quinoid and benzoid ring vibrations, thereby indicating the oxidation state of emeraldine salt PANI. A strong band observed at $1139.85 \mathrm{~cm}^{-1}(\mathrm{y}=0.5), 1140 \mathrm{~cm}^{-1}(\mathrm{y}=0.3)$, and1143.71 cm $\mathrm{cm}^{-1}(\mathrm{y}=0.1)$, described as the electronic-like band and is considered to be a measure of the degree of delocalization of electrons and thus it is characterized peak of PANI.The $\mathrm{C}-\mathrm{N}$ stretching in the neighborhood of quinoid ring, $\mathrm{C}-$ $\mathrm{N}$ stretch of secondary aromatic amine, the aromatic $\mathrm{C}-\mathrm{H}$ in plane bending modes is usually observed in the spectra of the three samples. The spectra of PTSA doped PANI samples confirmed the presence of dopant in the PANI.The X-ray diffraction pattern obtained for the three samples of PTSA doped PANI shows a typical emeraldine salt (conducting). The high angle X-ray scattering peaks observed in the X-ray diffraction pattern of PANI-PTSA shows significant crystallization of PANI due to protonation. The initial electrical conductivity was observed around $10^{-2} \mathrm{~S} \mathrm{~cm}^{-1}$ for PANI-(PTSA) $0_{0.5}, 10^{-3} \mathrm{~S} \mathrm{~cm}^{-1}$ for PANI-(PTSA) 0.3 and $10^{-5} \mathrm{~S} \mathrm{~cm}^{-1}$ for PANI$(\mathrm{PTSA})_{0.1}$, at $35^{\circ} \mathrm{C}$. However, the temperature dependence of electrical conductivity of PTSA doped PANI (in all the three doping levels) increases with increase of temperature, which suggests its thermal stability in the presence of oxygen. Also the conductivity measurement reveals that the PTSA doped PANI behaves like a semiconductor on comparison with standard values of electrical conductivity for traditional semiconductors. 


\section{Acknowledgements}

We are thankful to the Karpagam University for giving the facilities for the preparation of the samples. Also, we are thankful to Dr. Premnazeer, Principal of Islamiah College, Vaniambadi for his help and fruitful discussions.

\section{References}

[1] H. Letheby. J. Am. Chem. Soc. 15,161 (1862).

[2] Handbook of Organic Conductive Molecules and Polymers: Vol. 2. Conductive Polymers: Synthesis and Electrical Properties. Edited by H.S.Nalwa.

[3] J. C. Chiang, A. G. MacDiarmid, Synth. Met.13 (1986) 193.

[4] E. T. Kang, K. G. Neoha, K. L. Tan, Prog. Polym.Sci. 23 (1998) 277.

[5] S. Shreepathi, R. Holze, Chem. Mater. 17 (2005) 4078.

[6] Y. Furukawa, F. Ueda, Y. Hyodo, I. Harada, T. Nakajima, T. Kawagoe, Macromolecules 21 (1998) 1297.

[7] K. G. Conroy, C. B. Breslin, Electrochim. Acta 48 (2003) 721.

[8] J. E. Mark, In Polymer data Handbook, Oxford University, 1999.

[9] Ratheesh R and K.Viswanathan, 'Calorimetric and Thermogravimetric studies in Para Toluene Sulphonic Acid doped polyaniline', Int.J.Sci.Res.2(11), 466-468, 2013.

[10] Umesh.swaware surash s. Vmare, Rective and functional polymers 65 (2005) 343-350.

[11] A. Mirmohseni, G.G Wallace, Polymer 44 (2003) 3523-3528

[12] S. Quillard, G. Lauran, S. Lefrant, A.G Macmirand, Vibrational analysis of polyaniline: A comparative study of leucoemeraldine, emeraldine, and pernigraniline bases, Phys. Rev. B, vol. 50, pp. 12496-12512, 1994

[13] H. Zengin, W. Zhou, J. Jin, R. Czerw, D.W. Smith, Jr, L.Echegoyen, D.L Carroll, S.H. Foulgar, J. Ballato, Carbon nanotube doped polyaniline, Adv. Mate. Vol. 14, pp.1481-1483, 2002 